\title{
Operational Tomography: Fitting of Data Patterns
}

\author{
J. Řeháček, ${ }^{1}$ D. Mogilevtsev, ${ }^{2,3}$ and Z. Hradil ${ }^{1}$ \\ ${ }^{1}$ Department of Optics, Palacky University, 17. listopadu 12, 77146 Olomouc, Czech Republic \\ ${ }^{2}$ Institute of Physics, Belarus National Academy of Sciences, F. Skarina Avenue 68, Minsk 220072, Belarus \\ ${ }^{3}$ Instituto de Física, UNICAMP, CP 6165, Campinas-SP, 13083-970, Brazil
}

(Received 19 February 2010; published 29 June 2010)

\begin{abstract}
We build an operational scheme for the quantum state reconstruction based on the fitting of data patterns. Each data pattern corresponds to the response of the measurement setup to a predefined reference state. The set of data patterns can be measured experimentally in the calibration stage preceding to the reconstruction. The quorum of reference states plays the role of a positive operator valued measure in terms of which the reconstruction is done. As the main advantage, the procedure is free of notorious problems with projections into non-normalizable quadrature eigenstates, infinite dimensionality, ill-posed inversion, or imperfect detection.
\end{abstract}

PACS numbers: 03.65.Wj, 42.50.Lc

According to the pragmatic interpretation, the quantum theory is, first of all, the theory of measurement. All predictions must be phrased in terms of measurable quantities regardless of how abstract the structures used to describe the quantum features are. This is also the case for a quantum state reconstruction. Though its purpose is to identify the quantum state of the measured system, it may also be seen as the relationship between past and future observations of the same system. A quantum state is only an oxymoron for information about any possible future measurement encoded comprehensively into a convenient theoretical structure. Accepting this interpretation, any reconstruction procedure consists of classical data processing followed by quantum interpretation, which guarantees that any future prediction based on the original measurement must obey quantum rules, such as various uncertainty relations.

In this Letter we will elaborate on this pragmatic interpretation exploring the common roots of quantum tomography and classical data processing. Quantum interpretation will be postponed up to the very last moment where one wants to apply the inferred information to future quantum predictions. In this way we shall guide ourselves by analogies with classical optics, for example, by the analysis of a blurred image registered by a CCD camera, provided the optical response function is known. This approach offers significant advantages for a practical implementation of the reconstruction procedure. Indeed, the existing reconstruction methods are critically sensitive to such parameters as the detection efficiency, dimensionality of the problem, or the accuracy of the measurement [1]. The proposed procedure is free of these problems since those parameters are automatically accounted for in the data patterns in analogy with the optical response function.

Let us first detail the scheme of the classical image processing [2]. Though there are many options for mathematical implementation of the deconvolution procedure, all of them share some common features. The blurred image is decomposed into the superposition of elementary sources, the response function of which is supposed to be known. In classical optics, this formulation is usually done in the context of the coherent or incoherent image processing. Here, due to the analogies with quantum mechanics, we should go beyond this traditional approach and consider partially coherent signals as well. Let us denote $U\left(x_{1}\right)$ and $U\left(x_{2}\right)$ the amplitudes of an object and the corresponding image by a linear optical system. Only one dimension is considered here, for simplicity. Because of linearity, the transformation between the object and image planes can be expressed in the form of a superposition integral $U\left(x_{2}\right)=$ $\int U\left(x_{1}\right) h\left(x_{2}, x_{1}\right) d x_{1}$, where $h$ is the amplitude response function of the system. Considering the standard quadratic behavior of detectors in the optical domain and possibly stochastic character of optical fields, we get for the detected signal

$$
I\left(x_{2}\right)=\iint\left\langle U\left(x_{1}\right) U^{*}\left(x_{1}^{\prime}\right)\right\rangle h\left(x_{2}, x_{1}\right) h^{*}\left(x_{2}, x_{1}^{\prime}\right) d x_{1} d x_{1}^{\prime},
$$

where we denote $\langle\ldots\rangle$ averaging over those stochastic variables. Notice that the detected intensity is linear with respect to the mutual coherence matrix $G\left(x_{1}, x_{1}^{\prime}\right)=$ $\left\langle U\left(x_{1}\right) U^{*}\left(x_{1}^{\prime}\right)\right\rangle$. Now we are ready to continue with the deconvolution step.

The purpose of the deconvolution, or reconstruction, is to analyze intensity $I\left(x_{2}\right)$ corresponding to an unknown object coherence matrix $G\left(x_{1}, x_{1}^{\prime}\right)$. The reconstruction $\hat{G}$ will be given in terms of reference objects $G_{\xi}$, for which the intensity patterns $I_{\xi}$ are available:

$$
\hat{G}=\sum_{\xi} a_{\xi} G_{\xi} .
$$

Since the detected intensities Eq. (1) are linear in $G$, the coefficients $a_{\xi}$ can be obtained by fitting intensity patterns $I_{\xi}$. That is, we search for a linear combination of intensity patterns 


$$
\hat{I}=\sum_{\xi} a_{\xi} I_{\xi},
$$

which is as close as possible to the actually detected intensity $I$. The same set of fitting coefficients then provides the reconstruction of the object intensity via Eq. (2). More rigorously, the reconstruction procedure can be formulated as a multidimensional minimization of a distance between the linear combination of data patterns and target data. The simple and robust least-squares fit [3] minimizing the functional

$$
E\left(\left\{a_{\xi}\right\}\right)=\sum_{i}\left[I\left(x_{i}\right)-\hat{I}\left(x_{i}\right)\right]^{2}
$$

can be adopted for this purpose. Summation is done over points in the image plane. Denoting $J_{\xi}=\sum_{i} I_{\xi}\left(x_{i}\right) \hat{I}\left(x_{i}\right)$ and $M_{\eta, \xi}=\sum_{i} I_{\eta}\left(x_{i}\right) I_{\xi}\left(x_{i}\right)$, the least-squares fit can be cast in the matrix form $\mathbf{a}=\mathbf{M}^{-\mathbf{1}} \mathbf{J}$.

Notice that this approach is strictly operational in the sense that the result is determined by the measured data without any need to know the actual amplitude response function of the optical system. The only assumption we make is that the measurement is consistent, i.e., does not change in the course of the experiment. Considering the optical system as a part of detection, we may say that faithful reconstruction by data pattern fitting is possible without ever knowing what physical quantities have actually been measured-only the outcomes of these measurements matter. This feature brings about many practical advantages. For example, the result of reconstruction is not sensitive to the presence of systematic measurement errors.

Before closing the motivating example of image processing, let us discuss the issue of positivity of the reconstructed coefficients $a_{\xi}$. Provided all reconstructed coefficients are non-negative, the solution $\hat{G}$ is a sum of weighted reference coherence matrices $G_{\xi}$. This guarantees that the reconstructed coherence matrix is positive semidefinite in correspondence with the fact that the diagonal elements (for $x_{1}=x_{1}^{\prime}$ ) and so the eigenvalues of $\hat{G}$ are intensities and hence must be non-negative. The second possibility is that some reconstructed coefficients are negative. In this case, the positive semidefiniteness of the reconstructed coherence matrix must be verified, and provided this test fails, negative spectral components of $\hat{G}$ must be filtered out in order to ensure that all future predictions based on the reconstruction are consistent.

The theory of image processing based on the data patterns given above can be directly translated into the language of quantum theory. Indeed, the coherence matrix is nothing other than a position representation of the density matrix, $G\left(x, x^{\prime}\right)=\left\langle x|\rho| x^{\prime}\right\rangle$. Generalization is straightforward. Let us denote $G_{j k}=\langle j|\rho| k\rangle$ a particular representation of a density matrix describing the measured quantum system and replace the detection of the optical signal by a set of projective measurements $\left|y_{i}\right\rangle, h_{j i}=\left\langle j \mid y_{i}\right\rangle$ performed on the quantum system. Then quantum predictions given by the projection postulate $p_{i}=\left\langle y_{i}|\rho| y_{i}\right\rangle$, or, more generally, by $p_{i}=\operatorname{Tr}\left(\rho \Pi_{i}\right)$, where $\Pi_{i}$ are the elements of a positive operator valued measure (POVM), can be seen as a discrete version of the optical processing formula (1).

The proposed quantum reconstruction protocol follows closely the deconvolution procedure of the classical image processing. First, a set of reference states $\rho_{\xi}$ is measured and data patterns $f_{i}^{\xi}$ sampling probabilities $p_{i}^{\xi}$ are registered. The same set of measurements is applied to the unknown signal state $\rho$ with the result $f_{i}$. After that the reconstruction is obtained as the linear combination of the reference states

$$
\hat{\rho}=\sum_{\xi} a_{\xi} \rho_{\xi}
$$

minimizing the distance between the signal data and weighted patterns

$$
E\left(\left\{a_{\xi}\right\}\right)=\sum_{i}\left(f_{i}-\sum_{\xi} a_{\xi} f_{i}^{\xi}\right)^{2} .
$$

Finally, reconstruction errors may be estimated, see, e.g., [4], and used for discriminating relevant details from possible artefacts due to a limited number of reference states or statistical noise.

The proposed procedure based on fitting data patterns keeps all the good features of a conceptually similar image processing discussed above. In particular, the input quantum state can be reconstructed without having any knowledge about the measurement apparatus. The issue of positivity of the reconstructed coefficients discussed above also appears in the quantum formulation. Simple ad hoc regularization can be done by projecting the reconstructed $\hat{\rho}$ into a subspace, where it is semidefinite positive, e.g., by setting the negative eigenvalues of $\hat{\rho}$ to zero and renormalizing. This subspace would define the effective field of view of the tomography measurement. Such treatment is however rather arbitrary and not optimal. More rigorously, the operational tomography can be formulated as a semidefinite convex program [5],

$$
\operatorname{minimize} E\left(\left\{a_{\xi}\right\}\right) \quad \text { s.t. } \sum_{\xi} a_{\xi}=1, \quad \sum_{\xi} a_{\xi} \rho_{\xi} \geq 0,
$$

which can be solved by standard tools of convex optimization. It is worth mentioning that the positivity constraint imposed by quantum theory is, in fact, the very quantum part of the whole reconstruction procedure. Indeed, the fitting (5) deals with purely classical objects $f_{i}$ and $f_{i}^{\xi}$. In this sense, quantum interpretation of the measured object is relegated to the very last moment- the moment, when it is required that the predictions about future measurements based on the outcomes of past measurements be consistent with the postulates of quantum theory.

There are two issues which influence heavily the performance of the reconstruction, namely, the quorum of reference states and the measurement device producing 
the corresponding data patterns. The tomography setup should provide sufficiently rich data structure making faithful fitting of unknown input states possible. In general, larger sets of reference states are expected to yield better results as long as their data patterns can still be discriminated in the experiment. We also note that any particular choice of reference states is a commitment for an experimenter that such states can be generated on demand and their corresponding data patterns can be detected.

Let us turn to specific examples and discuss all stages outlined above in detail, considering optical homodyne tomography based on the measurement of rotated quadrature operators [6-8]. First we will clarify the choice of reference states. Quadrature eigenstates are not a good option here for they cannot be generated (even approximately) with the present technology. The most simple and feasible quorum of reference states in quantum optics seems to be a discrete set of coherent states $\rho_{\xi}=\left|\alpha_{\xi}\right\rangle \times$ $\left\langle\alpha_{\xi}\right|$ generated by a laser, whose complex amplitudes $\alpha_{\xi}$ can be manipulated by controlling two parameters-intensity and phase. This can be done by attenuating and phase shifting the laser beam. Such precise reference states have already been used for experimental tomography of quantum detectors [9].

When the result of reconstruction (4) is decomposed into coherent states, the coefficients $a_{\xi}$ appearing in this decomposition play a role analogous to the GlauberSudarshan representation already adopted for the complete characterization of quantum optical processes [10]. However, there are important differences here. The set of coherent states is overcomplete and not all of them are needed for representing the given state [11], or a given operator provided that projection into coherent states is used as an operator basis. Consequently, even a coherent state can be represented as a superposition with negative coefficients for a discrete quorum of other coherent states.

This is illustrated in Fig. 1, where three states of interest - a coherent state, a single-photon state, and a superposition of two coherent states-are represented by a quorum of 361 coherent states evenly distributed in the phase space. For each signal state, the coefficients $a_{\xi}$ are shown in panels (b)-(d). It turns out that in all three cases the fidelities of coherent-state representations [Eq. (4)] exceed 0.99999. Although the optimization of the set of reference states with respect to particular tomography setup may be a difficult task, a simple "rule of thumb" can be formulated that can be used to check whether the chosen set of coherent states provides a reasonably accurate description of permissible signal states: (i) the signal state should be localized within the region of phase space spanned by reference states, and (ii) the set of reference states should be dense enough "to feel" the fine features of the signal state. In practice, suitability of a given set of reference states should always be confirmed by evaluating estimation errors [4]. Quite interestingly, as the simulations show, introducing more classical noisy states in the set of (a)

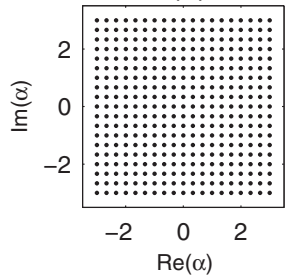

(c)

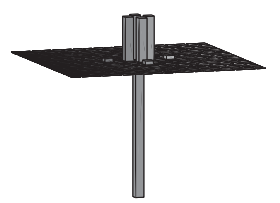

(d)

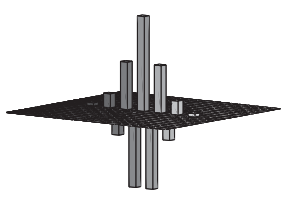

FIG. 1. A quorum of reference coherent states $\rho_{\xi}$ with regularly spaced amplitudes (a) and their coefficients $a_{\xi}$ appearing in the decomposition Eq. (4) of the coherent state with the amplitude $\alpha=0.763$ (b), the single-photon Fock state (c), and the Schrödinger-kitten state $|\alpha=0.763\rangle+|\alpha=-0.763\rangle$ (d).

reference coherent states can improve fitting of highly nonclassical states. For example, adding just one thermal state with 5 photons on average has a similar effect as doubling the number of coherent reference states. The optimal choice of the quorum of reference states is far from trivial and this question deserves further research.

For the purpose of numerical simulations, the inefficient homodyne detection can be described by a set of POVM elements $\Pi_{q}, q=X \exp (i \theta)$ [1]. Simulated data patterns obtained by sampling the detection probabilities $p(X, \theta)=$ $\operatorname{Tr}\left[\rho_{\xi} \Pi_{q}\right]$ are shown in Fig. 2 for three particular reference

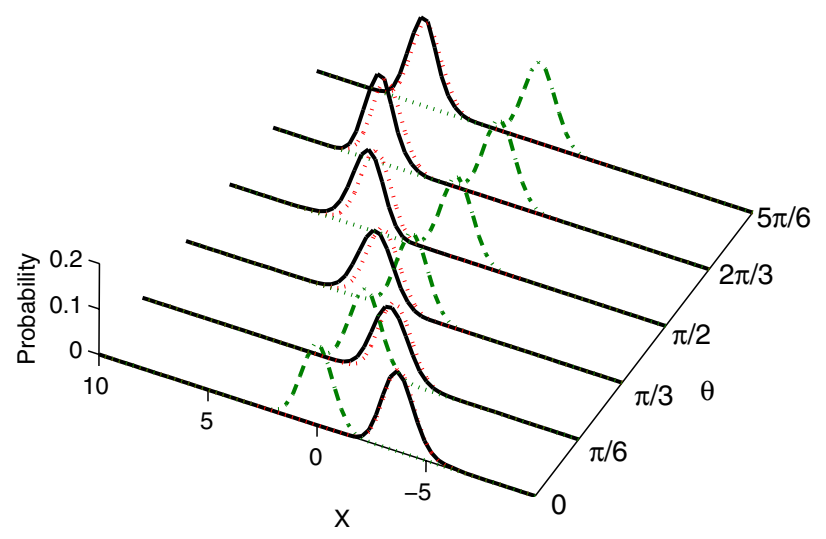

FIG. 2 (color online). Examples of data patterns generated by homodyne measurement for the vacuum reference state (green dash-dotted line), and two coherent reference states of amplitudes $\alpha=-3-3 i$ (black solid line) and $\alpha=-3-2.8421 i$ (red dotted line). In the figure, $x$ and $\theta$ denote the measured quadrature value and the phase of the local oscillator, respectively. In total, 120000 measurements were simulated for each quadrature and the outcomes were grouped in $j=1, \ldots, 64$ bins spanning the interval of $X_{j} \in[-10,10]$. Quantum efficiency $\nu=0.8$ of the homodyne detection was used in the simulation. 
(a)

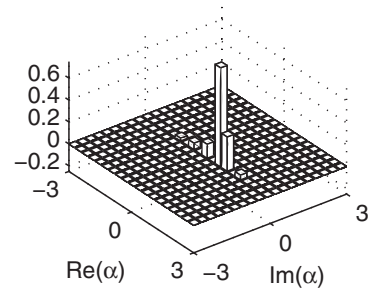

(c)

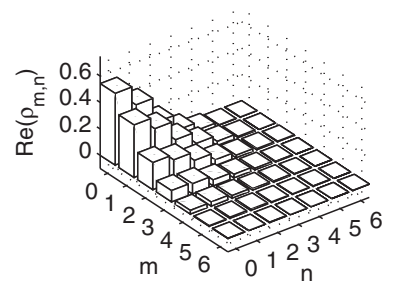

(b)

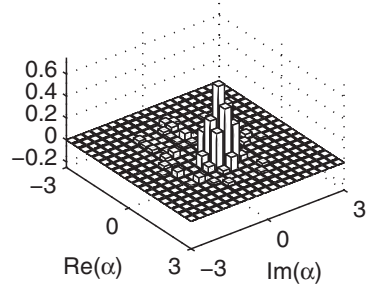

(d)

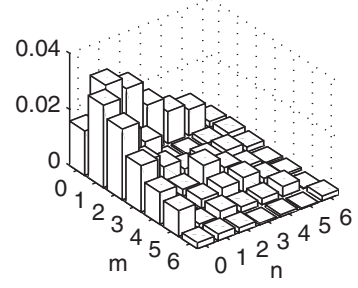

FIG. 3. Reconstruction of the coherent state of amplitude $\alpha_{\text {true }}=0.763$ from simulated homodyne measurements by fitting data patterns with the least-squares method (5). Parameters of the homodyne setup are the same as those in Fig. 2. Panels (a) and (b) show the true and reconstructed coefficients $a_{\xi}$. The set of reference states is the same as in Fig. 1(a). Panels (c) and (d) show the corresponding reconstructed density matrix elements $\rho_{m n}$ in the Fock basis and their respective errors.

states: the vacuum state and two coherent states taken as the pair of neighbors in the bottom right corner of the reference grid in Fig. 1(a). Notice that data patterns of the two coherent states are barely distinguishable, suggesting that the reference grid in Fig. 1(a) provides sufficient resolution in the phase space.

An example of a reconstruction of a coherent state by data patterns fitting is shown in Fig. 3. The same set of reference states as in Fig. 1(a) was used. For each reference state a data pattern was generated by simulating homodyne detection for six different phases of local oscillator as in Fig. 2. As seen, the estimated coefficients $x_{j}$ are close to the exact ones. For the chosen set of parameters the fidelity of the reconstructed state exceeds 0.95 .

Operational tomography presented in this Letter represents a full fledged alternative to standard tomography schemes. As the main advantage, the operational approach to tomography does not hinge upon having precise knowledge about the measurement performed on the physical system we want to characterize. As we have shown, this problem can be avoided by characterizing the measurement apparatus by patterns detected for a set of referenced states and matching the signal to those patterns by a leastsquares fitting, where the constraints of quantum mechanics on the reconstructed state are accommodated by either a projection into a proper subspace or incorporated directly into the fitting procedure.

Loosely speaking, while the standard tomography attempts to solve the problem in the fixed operator basis of known POVM elements [12-14], the proposed operational

tomography is doing the same in the frame specified by detected data patterns. Since operational tomography does not require knowledge of the measurement apparatus and all information used for the reconstruction can always be cast in the form of measured data patterns, it is free of any assumption that cannot be verified experimentally and hence should be less prone to systematic errors than standard methods should. Remarkably, the procedure does not split into separate calibration and estimation stages, but handles all the data in equal manner-data patterns corresponding to an unknown state are fitted to respective patterns of reference quantum states. Operational quantum tomography is therefore fully analogous to image processing techniques. We hope that the proposed concept will be found interesting and inspiring for diagnostics of nonclassical effects.

This work was supported by the Czech Ministry of Education, Project No. MSM6198959213, and the Czech Ministry of Industry and Trade, Project No. FR-TI1/364 (J. R. and Z. H.), as well as the EU under EQUIND, project of 6FP IST-034368, BRFFI of Belarus, and FAPESP of Brazil (D. M.).

[1] Quantum States Estimation, edited by M. G. A. Paris and J. Řeháček Lect. Notes Phys. Vol. 649 (Springer, Berlin Heidelberg, 2004).

[2] R. G. Gonzalez and R.E. Woods, Digital Image Processing (Prentice-Hall, Englewood Cliffs, NJ, 2002), 2nd ed.

[3] J. Wolberg, Data Analysis Using the Method of Least Squares: Extracting the Most Information from Experiments (Springer, New York, 2005).

[4] J. Řeháček, D. Mogilevtsev, and Z. Hradil, New J. Phys. 10, 043022 (2008).

[5] S. Boyd and L. Vandenberghe, Convex Optimization (Cambridge University Press, Cambridge, England, 2009).

[6] D. T. Smithey, M. Beck, M. G. Raymer, and A. Faridani, Phys. Rev. Lett. 70, 1244 (1993).

[7] A. Ourjoumtsev, R. Tualle-Brouri, and P. Grangier, Phys. Rev. Lett. 96, 213601 (2006).

[8] J. S. Neergaard-Nielsen, B. M. Nielsen, C. Hettich, K. Molmer, and E. S. Polzik, Phys. Rev. Lett. 97, 083604 (2006).

[9] J. S. Lundeen et al., Nature Phys. 5, 27 (2009).

[10] M. Lobino, D. Korystov, C. Kupchak, E. Figueroa, B. C. Sanders, and A. I. Lvovsky, Science 322, 563 (2008).

[11] A. M. Perelomov, Theor. Math. Phys. 6, 156 (1971); see also arXiv:math-ph/0210005v1.

[12] K. Banaszek, G. M. D’Ariano, M. G. A. Paris, and M. F. Sacchi, Phys. Rev. A 61, 010304(R) (1999).

[13] D. F. V. James, P. G. Kwiat, W. J. Munro, and A. G. White, Phys. Rev. A 64, 052312 (2001).

[14] Z. Hradil, D. Mogilevtsev, and J. Řeháček, Phys. Rev. Lett. 96, 230401 (2006); D. Mogilevtsev, J. Řeháček, and Z. Hradil, Phys. Rev. A 75, 012112 (2007). 